PROCEEDINGS OF THE

AMERICAN MATHEMATICAL SOCIETY

Volume 127, Number 6, Pages 1827-1836

S 0002-9939(99)04809-1

Article electronically published on February 18, 1999

\title{
ACCELERATIONS OF RIEMANNIAN QUADRATICS
}

\author{
LYLE NOAKES
}

(Communicated by Christopher Croke)

\begin{abstract}
A Riemannian corner-cutting algorithm generalizing a classical construction for quadratics was previously shown by the author to produce a $C^{1}$ curve $p_{\infty}$ whose derivative is Lipschitz. The present paper takes the analysis of $p_{\infty}$ a step further by proving that it possesses left and right accelerations everywhere. Two-sided accelerations are shown to exist on the complement of a countable dense subset $D$ of the domain. The results are shown to be sharp in the following sense. For almost any scaled triple in Euclidean space there is a Riemannian perturbation of the Euclidean metric such that the two-sided accelerations of the resulting curve $p_{\infty}$ exist nowhere in $D$.
\end{abstract}

\section{BACKGROUND IN BRIEF}

A very detailed description of the construction of the Riemannian quadratics is given in [11], but the following summary is enough for the present paper to be read independently. Let $\langle$,$\rangle be a Riemannian metric on an open subset V$ of $\mathbb{R}^{n}$, possibly realised as a coordinate chart of a more general Riemannian manifold. Let $V$ be geodesically convex in the sense that any two points in $V$ are joined by a geodesic segment, unique up to reparameterization and minimal. Let $U$ be another open subset of $\mathbb{R}^{n}$ whose closure is compact and contained in $V$.

Let $d$ be the metric on $U$ defined by the Riemannian distance. Then $d\left(x_{a}, x_{b}\right)$ is bounded for all $x_{a}, x_{b} \in U$. For $a<b \in \mathbb{R}$ let $C[a, b]$ be the complete metric space of continuous curves $\omega:[a, b] \rightarrow U$ with respect to the uniform metric $d_{U}$ where

$$
d_{U}\left(\omega, \omega^{\prime}\right)=\operatorname{Max}_{t \in[a, b]} d\left(\omega(t), \omega^{\prime}(t)\right) .
$$

The Christoffel transformations

$$
\Gamma_{x}: \mathbb{R}^{n} \times \mathbb{R}^{n} \rightarrow \mathbb{R}^{n}
$$

are also bounded for $x \in U$.

Because $U$ is convex we can define for any $x_{a}, x_{b} \in U$ the midpoint $M\left(x_{a}, x_{b}\right) \in$ $U$ to be $\omega((a+b) / 2)$ where $\omega:[a, b] \rightarrow U$ is the minimal geodesic from $x_{a}$ to $x_{b}$. A Riemannian scaled triple is a quadruple $Y=\left(y_{0}, y_{1}, y_{2}, h\right) \in U^{3} \times \mathbb{R}_{+}$ where $y_{0}, y_{1}, y_{2}$ are the vertexes of $Y$ and $h$ is the scale. The fundamental polygon $p:[0,2 h] \rightarrow U$ of $Y$ is the track sum of the geodesic segments joining $y_{0}, y_{1}$ and $y_{1}, y_{2}$, parameterized by $[0, h]$ and $[h, 2 h]$ respectively. Using the midpoint map

Received by the editors December 7, 1996 and, in revised form, June 11, 1997.

1991 Mathematics Subject Classification. Primary 53B20, 53B99; Secondary 41A15, 41A29, $41 \mathrm{~A} 99$

Key words and phrases. Geodesic, parallel translation, corner-cutting.

(C)1999 American Mathematical Society 
$M: U \times U \rightarrow U$, a splitting of $Y$ into its left triple $Y^{L}$ and right triple $Y^{R}$ are defined as follows.

Definition 1. Let $y_{3}=M\left(y_{0}, y_{1}\right), y_{4}=M\left(y_{1}, y_{2}\right)$ and $y_{5}=M\left(y_{3}, y_{4}\right)$. Then $Y^{L}$ is the scaled triple $\left(y_{0}, y_{3}, y_{5}, h / 2\right)$ and $Y^{R}=\left(y_{5}, y_{4}, y_{2}, h / 2\right)$.

Splitting can also be applied to $Y^{L}$ and then $Y^{R}$, producing scaled triples

$$
Y^{L L}, Y^{L R}, Y^{R L}, Y^{R R}
$$

of scales $h / 4$. Continuing, after $m$ iterations we obtain, for every word $w$ of length $m$ in the symbols $L, R$, a scaled triple $Y^{w}$ of scale $h / 2^{m}$ called descendants of $Y$ in generation $m$. Writing the $Y^{w}$ in dictionary order, the track-sum $p_{m}$ of their fundamental polygons turns out to have a smoother appearance than the fundamental polygon $p$. Indeed the main result of [11] is

Theorem 1. The sequence $\left\{p_{m}: m \geq 1\right\}$ converges uniformly in $C[0,2 h]$ to a curve $p_{\infty} \in C[0,2 h]$ with the properties

1. $p_{\infty}$ is differentiable on $(0,2 h)$,

2. $p_{\infty}$ is right-differentiable at 0 ,

3. $p_{\infty}$ is left-differentiable at $2 h$,

4. $\dot{p}_{\infty}$ is Lipschitz.

For the classical quadratic algorithm, $\langle$,$\rangle is the Euclidean inner product and p_{\infty}$ is well-known to be a quadratic polynomial curve. However, other generalizations of the classical algorithm produce curves with pathological properties [12], [4], [5], [6], [7], [8], [1]. The present Riemannian generalization turns out to be both regular and pathological.

Let $D \subset[0,2 h]$ be the countable dense subset consisting of multiples of $h$ by dyadic rationals. Except in the classical case it is rare for $p_{\infty}$ to be twice differentiable at points in $D$. Interestingly, although Theorem 1 says $p_{\infty}$ is $C^{1}$ everywhere, it is really only at points in $D$ that this seems plausible. So we might expect higher derivatives of $p_{\infty}$ to be better behaved on $D$ than elsewhere: exactly the opposite is true. In fact the main result of the present paper is

Theorem 2. 1. $\dot{p}_{\infty}$ is left-differentiable on $(0,2 h]$.

2. The left-acceleration $\ddot{p}_{\infty}$ - is left-continuous.

3. $\dot{p}_{\infty}$ is right-differentiable on $[0,2 h)$.

4. The right-acceleration $\ddot{p}_{\infty}+$ is right-continuous.

5. $\dot{p}_{\infty}$ is differentiable on the complement of $D$ in $[0,2 h]$.

The proof of Theorem 2 is carried out in two stages. First, candidates for the one-sided covariant derivatives of the velocity field $\dot{p}_{\infty}$ are constructed as limits of sequences of functions $\alpha_{m \pm}:[0,2 h] \rightarrow \mathbb{R}^{n}$. The $\alpha_{m \pm}$ are themselves constructed from accelerations of descendants of the scaled triple $Y$ together with the geometric operation of parallel translation. Inheritance properties of accelerations of scaled triples lead to analytic results concerning the $\alpha_{m \pm}$ and their limits. The second step is to prove that the limits are in fact one-sided accelerations of $p_{\infty}$. The main ingredients are an inheritance property for accelerations of scaled triples, and the well-known relationship between geodesics and parallel translation.

The question of whether $\dot{p}_{\infty}$ has a two-sided derivative at points in $D$ may now be considered. For any Riemannian manifold there will always be special configurations of $y_{0}, y_{1}, y_{2}$ for which the answer is "yes". 
Example 1. Suppose that $y_{1}$ lies on the minimal geodesic $\omega$ : $[0,2 h]$ from $y_{0}$ to $y_{2}$. Each $p_{m}$ is obtained by preceding $\omega$ with a piecewise-linear function

$$
q_{m}:[0,2 h] \rightarrow[0,2 h],
$$

and the sequence $\left\{q_{m}: m \geq 1\right\}$ converges uniformly to a quadratic function of the form

$$
q_{\infty}(t)=a t+(1-a) t^{2} /(2 h)
$$

where $\omega(a h)=y_{1}$. Then $p_{\infty}=\omega \circ q_{\infty}$ and is therefore $C^{\infty}$.

The answer is also "yes" when $U=\mathbb{R}^{n}$ with the Euclidean inner product, regardless of the scaled triple $Y$, because then $p_{\infty}$ is a quadratic polynomial curve. However, a small Riemannian perturbation can completely change this, as the following result shows.

Theorem 3. Let $y_{0}, y_{1}, y_{2}$ be non-colinear points in $\mathbb{R}^{n}$ with the Euclidean metric. Fix $h>0$. Then there is a Riemannian metric $\langle\rangle,, C^{\infty}$-close to the Euclidean inner product, with the following property. For the Riemannian scaled triple $Y=$ $\left(y_{0}, y_{1}, y_{2}, h\right)$ of $\left(\mathbb{R}^{n},\langle\rangle,\right), p_{\infty}$ has a two-sided derivative nowhere in $D$.

The proof is by direct construction and is given in $\S 4$.

\section{AcCelerations of triples}

The mesh $\mu(Y)$ of a scaled triple $Y=\left(y_{0}, y_{1}, y_{2}, h\right)$ is defined to be the larger of $d\left(y_{0}, y_{1}\right), d\left(y_{1}, y_{2}\right)$. Recall from [11] that $\mu\left(Y^{w}\right) \leq \mu(Y) / 2^{m}$ where $w$ is a word of length $m$ in the symbols $L, R$. The acceleration $\alpha(Y)$ of a scaled triple $Y$ is defined in $[11]$ as

$$
\left(\dot{\omega}_{12}(h)-\dot{\omega}_{01}(h)\right) /(2 h)
$$

where, for $i=0,1, \omega_{i i+1}:[i h,(i+1) h] \rightarrow U$ is the minimal geodesic from $y_{i}$ to $y_{i+1}$. Recall from [11] the following inheritance property of accelerations of scaled triples.

\section{Lemma 2.1.}

$$
\alpha\left(Y^{L}\right)=\alpha(Y)+O(1) \mu(Y)=\alpha\left(Y^{R}\right) .
$$

In a Riemannian setting the parallel translation of a vector based at $x_{a}$ to a vector based at $x_{b}$ depends on a choice of curve joining $a, b$. The curve needs to be $C^{1}$ and the velocity vector should satisfy a Lipschitz condition. Parallel translation is also defined along a continuous track-sum of such curves with finitely many summands. Parallel translation is transitive and preserves Riemannian inner products. More details can be found in introductory books on Riemannian geometry, for example [2]. For $m \geq 1$ accelerations of scaled triples can be used to define functions

$$
\alpha_{m-}, \alpha_{m+}:[0,2 h] \rightarrow \mathbb{R}^{n} .
$$

Roughly speaking, $\alpha_{m-}(s)$ is the parallel translation along $p_{m}$ to $p_{m}(s)$ of the acceleration of the nearest scaled triple to the left of $s$ in generation $m$. Replacing "left" by "right" describes $\alpha_{m+}(s)$. The formal definitions are as follows.

Definition A. 1. For $t \in\left(2(i-1) h / 2^{m}, 2 i h / 2^{m}\right]$ let $w$ be the $i$ th word of length $m$ in the symbols $L, R$. Then $\alpha_{m-}(t)$ is the parallel translation of $\alpha\left(Y^{w}\right)$ along $p_{m}$ from $p_{m}\left((2 i-1) h / 2^{m}\right)$ to $p_{m}(t)$. 
2. For $t \in\left[2(i-1) h / 2^{m}, 2 i h / 2^{m}\right)$ let $w$ be the $i$ th word of length $m$ in the symbols $L, R$. Then $\alpha_{m+}(t)$ is the parallel translation of $\alpha\left(Y^{w}\right)$ along $p_{m}$ from $p_{m}\left((2 i-1) h / 2^{m}\right)$ to $p_{m}(t)$.

3. Set $\alpha_{m-}(0)=\alpha_{m+}(0)$ and $\alpha_{m+}(2 h)=\alpha_{m-}(2 h)$.

From the definitions $\alpha_{m-}$ is left-continuous on $(0,2 h]$, and $\alpha_{m+}$ is right-continuous on $[0,2 h)$. Let $D_{m}=\left\{2 i h / 2^{m}: 1 \leq i \leq 2^{m}\right\}$. Then $\alpha_{m-}$ and $\alpha_{m+}$ agree on $[0,2 h]-D_{m}$ and so they are continuous except possibly at points in $D_{m}$. (Interestingly the left and right derivatives $\dot{p}_{m-}, \dot{p}_{m+}$ of the piecewise-geodesic $p_{m}$ are continuous at points in $D_{m}$ ).

Lemma 2.2. The sequences

$$
\left\{\alpha_{m-}: m \geq 1\right\} \quad \text { and } \quad\left\{\alpha_{m+}: m \geq 1\right\}
$$

converge uniformly with respect to the Euclidean norm on $\mathbb{R}^{n}$.

Proof. For $t \in\left[2(i-1) h / 2^{m}, 2 i h / 2^{m}\right), \alpha_{m+}(t)-\alpha_{m+1+}(t)$ is the difference after parallel translation along $p_{m}$ between

$$
\alpha\left(Y^{w}\right) \text { and } \alpha\left(\left(Y^{w}\right)^{L}\right), \quad \text { or } \quad \alpha\left(Y^{w}\right) \text { and } \alpha\left(\left(Y^{w}\right)^{R}\right)
$$

where $w$ is the $i$ th word of length $m$ in the symbols $L, R$.

In either case the norm of the difference is $O(1) \mu\left(Y^{w}\right)=O(1) \mu(Y) / 2^{m}$ by Lemma 2.1 applied to the scaled triple $Y^{w}$. The same holds when $t=2 h$. So $\left\{\alpha_{m+}: m \geq 1\right\}$ is Cauchy and therefore convergent. A similar argument applies to the sequence of left-accelerations and the lemma is proved.

Let $\alpha_{\infty-}, \alpha_{\infty+}$ be the limits of the respective sequences in Lemma 2.2. Because $\alpha_{m-} \mid(0,2 h]$ is left-continuous and $\alpha_{m+} \mid[0,2 h)$ is right-continuous for $m \geq 1$ we obtain

Lemma 2.3. $1 . \alpha_{\infty-} \mid(0,2 h]$ is left-continuous.

2. $\alpha_{\infty+} \mid[(0,2 h)$ is right-continuous.

Lemma 2.4. If $s \notin D_{m}$, then

$$
\alpha_{\infty+}(s)=\alpha_{\infty-}(s)+O(1) \mu(Y) / 2^{m} .
$$

Proof. Because $s \notin D_{m}, s \in\left(2(i-1) h / 2^{m}, 2 i h / 2^{m}\right)$ for some $1 \leq i \leq 2^{m}$. For $r \geq 1, \alpha_{m+r-}(s)=\alpha\left(Z_{-}\right)+O(1) \mu(Y) / 2^{m}$ and $\alpha_{m+r+}(s)=\alpha\left(Z_{+}\right)+O(1) \mu(Y) / 2^{m}$ where $Z_{+}, Z_{-}$are descendants in generation $r$ of $Y^{w}$ (possibly the same descendant). Here $w$ is the $i$ th word in the symbols $L, R$. By Lemma 2.1

$$
\alpha\left(Z_{+}\right)=\alpha\left(Y^{w}\right)+O(1) \mu(Y) / 2^{m}=\alpha\left(Z_{-}\right)
$$

and this proves the lemma.

Let $D_{\infty}=\bigcup_{m \geq 1} D_{m}$. By Lemma 2.4, $\alpha_{\infty-}$ and $\alpha_{\infty+}$ agree on the complement of $D_{\infty}$.

Lemma 2.5. $\alpha_{\infty-}$ and $\alpha_{\infty+}$ are continuous at any point in the complement of $D_{\infty}$

Proof. Because $s \notin D_{\infty}$, given any $m \geq 1$ and any $t$ sufficiently near $s$, we have $t \notin D_{m}$ and therefore $\alpha_{m-}(t)=\alpha_{m+}(t)$. As in the proof of Lemma 2.4,

$$
\alpha_{m-}(t)-\alpha_{\infty-}(t)=O(1) \mu(Y) / 2^{m}=\alpha_{m+}(t)-\alpha_{\infty+}(t)
$$


and therefore

$$
\alpha_{\infty-}(t)-\alpha_{\infty+}(t)=O(1) \mu(Y) / 2^{m} .
$$

Similarly, $\alpha_{\infty-}(s)=\alpha_{\infty+}(s)$. Because $\alpha_{\infty_{-}}, \alpha_{\infty+}$ are left and right-continuous this proves the lemma.

Next we establish relationships between the functions

$$
\alpha_{\infty-}, \alpha_{\infty+}:[0,2 h] \rightarrow \mathbb{R}^{n}
$$

and the left and right covariant accelerations of the curve $p_{\infty}$ resulting from the Riemannian quadratic construction.

\section{Proof of Theorem 2}

Let $q:[a, b] \rightarrow U$ be $C^{1}$ where $\dot{q}$ is Lipschitz, and let $X_{t} \in \mathbb{R}^{n}$ be a vector based at $q(t)$. The parallel translation of $X_{t}$ along $q$ from $q(t)$ to $q(s)$ is denoted by $X_{t \rightarrow s}$. In particular, $\dot{q}$ may be regarded as a vector field defined along the curve $q$, so that $\dot{q}(t)$ is based at $q(t)$. We have two kinds of cases in mind. Firstly, $q$ might be $p_{\infty}$ and then $\dot{q}$ is Lipschitz according to Theorem 1. Secondly, $q$ might be a geodesic segment. Parallel translation along a track-sum of geodesics is defined by parallely translating along successive summands.

Definition C. 1 . The left covariant acceleration $\nabla_{\dot{q}(s)-} \dot{q}$ of $q$ at time $s \in(0,2 h]$ is defined to be

$$
\lim _{t \rightarrow s-}\left(\dot{q}(t)_{t \rightarrow s}-\dot{q}(s)\right) /(t-s)
$$

whenever the limit exists.

2. The right covariant acceleration $\nabla_{\dot{q}(s)+} \dot{q}$ of $q$ at time $s \in[0,2 h)$ is

$$
\lim _{t \rightarrow s+}\left(\dot{q}(t)_{t \rightarrow s}-\dot{q}(s)\right) /(t-s)
$$

when the limit exists.

A necessary and sufficient condition for $\dot{q}$ to be left- (respectively right-) differentiable at $s$ is that the left (respectively right) covariant acceleration of $q$ should exist at time $s$. A necessary and sufficient condition for the two-sided acceleration $\ddot{q}(s)$ to exist is that

$$
\nabla_{\dot{q}(s)-} \dot{q}=\nabla_{\dot{q}(s)+} \dot{q} .
$$

The reason for considering covariant accelerations instead of $\ddot{q}$ is that geometric constructions are most easily investigated using covariant objects. When $q=p_{\infty}$ it is by no means clear whether either of the covariant accelerations exist. A large part of the answer provided by Theorem 2 comes from the following result.

Lemma 3.1. 1. For $s \in(0,2 h]$

$$
\nabla_{\dot{p}_{\infty}(s)-} \dot{p}_{\infty}=\alpha_{\infty-}(s) .
$$

2. For $s \in[0,2 h)$

$$
\nabla_{\dot{p}_{\infty}(s)+} \dot{p}_{\infty}=\alpha_{\infty+}(s)
$$


Proof. Given $s \in[0,2 h)$ and $l \geq 1$ we have $s \in\left[2(k-1) h / 2^{l}, 2 k h / 2^{l}\right)$ for some $k$. Let $t>s$ lie in the same subinterval. Given $m>l$ let

$$
s \in\left(2(i-1) h / 2^{m}, 2 i h / 2^{m}\right] \text { and } t \in\left(2(j-1) h / 2^{m}, 2 j h / 2^{m}\right) .
$$

Then $i \leq j$. Because $s, t$ are separated by at least $j-i$ subintervals of length $2 h / 2^{m}$

$$
2(j-i) h / 2^{m} \leq t-s .
$$

Let $X_{-}$and $X_{+}$be the piecewise-continuous left and right velocity vector fields $\dot{p}_{m-}$ and $\dot{p}_{m+}$, defined along the piecewise geodesic curve $p_{m}$. When $u$ is an integer each restriction of $p_{m}$ to a subinterval of $[0,2 h]$ of the form $\left[(2 u-1) h / 2^{m}\right.$, $\left.(2 u+1) h / 2^{m}\right]$ is a geodesic. Because velocities of geodesics are translated parallely,

$$
X_{-(2 u+1) h / 2^{m} \rightarrow(2 u-1) h / 2^{m}}=X_{+(2 u-1) h / 2^{m}} .
$$

Therefore $X_{+t \rightarrow s}-\dot{p}_{m+}(s)$ can be written in the following form:

$$
\begin{gathered}
\left(X_{+t \rightarrow(2 j-1) h / 2^{m}}-X_{\left.-(2 j-1) h / 2^{m}\right)}\right)_{(2 j-1) h / 2^{m} \rightarrow s}+ \\
\left(X_{+(2 j-3) h / 2^{m}}-X_{-(2 j-3) h / 2^{m}}\right)_{(2 j-3) h / 2^{m} \rightarrow s}+ \\
\ldots \\
\left(X_{+(2 u+1) h / 2^{m}}-X_{-(2 u+1) h / 2^{m}}\right)_{(2 u+1) h / 2^{m} \rightarrow s}+ \\
\left(X_{+(2 u-1) h / 2^{m}}-X_{-(2 u-1) h / 2^{m}}\right)_{(2 u-1) h / 2^{m} \rightarrow s}+ \\
\cdots \\
\left(X_{+(2 i+1) h / 2^{m}}-X_{-(2 i+1) h / 2^{m}}\right)_{(2 i+1) h / 2^{m} \rightarrow s}+ \\
\left(X_{+(2 i-1) h / 2^{m} \rightarrow s}-\dot{p}_{m+}(s)\right) .
\end{gathered}
$$

Here every term is a parallel translation to $p_{m}(s)$ of a difference of translated velocities parameterized within a subinterval of width $2 h / 2^{m}$.

All but the first and last terms are scalar multiples by $2 h / 2^{m}$ of parallel translations to $p_{m}(s)$ of accelerations of the descendants

$$
Y^{1}, Y^{2}, \ldots, Y^{2^{m}}
$$

of the scaled triple $Y$. The sum of these intermediate terms is

$$
2 h / 2^{m} \sum_{i<u<j} \alpha\left(Y^{u}\right)_{(2 u-1) h / 2^{m} \rightarrow s}
$$

The first and last terms are small multiples of parallel translations $\alpha\left(Y^{j}\right)$ and $\alpha\left(Y^{i}\right)$, depending on the precise locations of $s$ and $t$. In any case

$$
X_{+t \rightarrow s}-\dot{p}_{m+}(s)-2 h / 2^{m} \sum_{i<u<j} \alpha\left(Y^{u}\right)_{(2 u-1) h / 2^{m} \rightarrow s}
$$

is bounded in norm by $O(1) \mu(Y) / 2^{m}$ according to Lemma 2.1 .

Recall that $t$ was chosen to lie in the subinterval $\left[2(k-1) h / 2^{l}, 2 k h / 2^{l}\right)$ containing $s$ where $l$ was given. Let $Z$ be the $k$ th descendant of $Y$ in generation $l$. Then the scaled triples

$$
Y^{i}, Y^{i+1}, \ldots, Y^{j}
$$

are descendants of $Z$ in generation $m-l$. So be Lemma 2.1 the accelerations

$$
\alpha\left(Y^{j}\right), \alpha\left(Y^{j-1}\right), \ldots, \alpha\left(Y^{i+1}\right), \alpha\left(Y^{i}\right)
$$


differ in norm by $O(1) \mu(Z)=O(1) \mu(Y) / 2^{l}$. Therefore

$$
\begin{aligned}
\left\|X_{+t \rightarrow s}-\dot{p}_{m+}(s)-(t-s) \alpha\left(Y^{i}\right)\right\| \\
\quad \leq\left\|X_{+t \rightarrow s}-\dot{p}_{m+}(s)-(j-i)\left(2 h / 2^{m}\right) \alpha\left(Y^{i}\right)\right\|+O(1) \mu(Y) / 2^{m} \\
\quad \leq(j-i+1)\left(2 h / 2^{m}\right) O(1) \mu(Y) / 2^{l}+O(1) \mu(Y) / 2^{m} \\
\quad=(t-s) O(1) \mu(Y) / 2^{l}+O(1) \mu(Y) / 2^{m}
\end{aligned}
$$

by (SEP). As $m \rightarrow \infty$ the inequality becomes

$$
\left\|\dot{p}_{\infty}(t)_{t \rightarrow s}-\dot{p}_{\infty}(s)-(t-s) \alpha_{\infty+}(s)\right\|=(t-s) O(1) \mu(Y) / 2^{l} .
$$

To complete the proof of part 2 of the lemma let $l \rightarrow \infty$. Part 1 follows from part 2 applied to the scaled triple $\left(y_{2}, y_{1}, y_{0}, h\right)$.

So the left and right covariant derivatives exist on $(0,2 h]$ and on $[0,2 h)$, respectively. The left covariant derivative is left-continuous and the right covariant derivative is right-continuous by Lemma 2.3. They also agree on the complement of $D_{\infty}$ by Lemma 2.4. Theorem 2 is proved.

\section{Proof of Theorem 3}

Let $Y$ be a scaled triple. We first deform the Euclidean metric so as to change the descendants $Y^{L}, Y^{R}$ of $Y$ in generation 1 while retaining the Euclidean metric on the convex hull of the vertices of $Y^{L}, Y^{R}$. Only the convex hull is relevant to further corner-cutting and so this kind of perturbation can be carried out with $Y^{L}$, $Y^{R}$ in place of $Y$, as in Lemma 4.2. The perturbation in Lemma 4.1 is chosen to create differences between the accelerations of $Y, Y^{L}, Y^{R}$.

Lemma 4.1. Let $Y=\left(y_{0}, y_{1}, y_{2}, h\right)$ be a Riemannian scaled triple for the Euclidean metric on $U \subseteq \mathbb{R}^{n}$ where $y_{0}, y_{1}, y_{2}$ are not colinear. Then there is a perturbation of the Euclidean metric to a Riemannian metric $\langle$,$\rangle with the following properties:$

(a) $\langle$,$\rangle is C^{\infty}$-close to Euclidean,

(b) $\langle$,$\rangle is Euclidean on the convex hull of y_{0}, y_{3}, y_{4}, y_{2}$ where

$$
y_{3}=M\left(y_{0}, y_{1}\right), \quad y_{4}=M\left(y_{1}, y_{2}\right)
$$

and midpoints are calculated using the Riemannian metric $\langle$,$\rangle ,$

(c) neither $y_{0}, y_{3}, y_{5}$ nor $y_{5}, y_{4}, y_{2}$ are colinear,

(d) $\alpha(Y), \alpha\left(Y^{L}\right), \alpha\left(Y^{R}\right)$ are distinct, where $Y^{L}, Y^{R}$ and their accelerations are calculated using $\langle$,$\rangle .$

Proof. Let $c=\left(3 y_{1}+y_{2}\right) / 4$. Because $y_{0}, y_{1}, y_{2}$ are not colinear there is an open ball $B(c, r)$ which does not intersect the segment $y_{0} y_{1}$. Without loss $0<r \leq\left\|y_{1}-y_{2}\right\| / 8$. Let $r$ be so small that $B(x, r)$ does not intersect the segment whose endpoints are $\left(y_{0}+y_{1}\right) / 2$ and $\left(y_{1}+y_{2}\right) / 2$.

Modify the Euclidean metric on $\mathbb{R}^{n}$ by inserting a ridge within $B(x, r / 2)$ whose axis $A$ is orthogonal to the segment $y_{1} y_{2}$. Flatten the ends of $A$ within $B(x, r)-$ $B(x, r / 2)$ so that the resulting Riemannian metric is Euclidean outside $B(x, r)$. Then

$$
y_{4}=a y_{1}+(1-a) y_{2} \quad \text { where } a \in(1 / 2,1)
$$

and $a$ is close to $1 / 2$ when the ridge has small height $\rho$. Choose $\rho>0$ so small that $y_{4}$ is very close to $\left(y_{1}+y_{2}\right) / 2$, namely so close that the segment $y_{3} y_{4}$ does not 
intersect $B(x, r)$. Here $y_{3}=M\left(y_{0}, y_{1}\right)=\left(y_{0}+y_{1}\right) / 2$ as with the Euclidean metric. Then (a), (b) are satisfied.

Since $y_{0}, y_{1}, y_{2}$ are not colinear neither are

$$
y_{0}\left(y_{0}+y_{1}\right) / 2, \quad\left(y_{0}+2 y_{1}+y_{2}\right) / 4
$$

nor

$$
\left(y_{0}+2 y_{1}+y_{2}\right) / 4, \quad\left(y_{1}=y_{2}\right) / 2, y_{2} .
$$

Now $y_{3}=\left(y_{0}+y_{1}\right) / 2$ and

$$
y_{4} \approx\left(y_{1}+y_{2}\right) / 2, \quad y_{5} \approx\left(y_{0}+2 y_{1}+y_{2}\right) / 4 .
$$

If $\rho>0$ is small enough these approximations ensure (c). To prove (d) note that

$$
y_{5}=M\left(y_{3}, y_{4}\right)=\left(y_{0}+(1+2 a) y_{1}+2(1-a) y_{2}\right) / 4
$$

and then

$$
\alpha\left(Y^{R}\right)-\alpha\left(Y^{L}\right)=2(1-2 a)\left(y_{1}-y_{2}\right) / h^{2} .
$$

Similarly, $\alpha\left(Y^{L}\right) \neq \alpha(Y) \neq \alpha\left(Y^{R}\right)$.

Next Lemma 4.1 is used to generate a sequence of perturbations of the Euclidean metric. Perturbations in generation $m+1$ are negligible in comparison with those in generation $m$, so that differences in accelerations in generation $m$ are not wiped out by subsequent perturbations. Then the main differences between accelerations of triples in generation $m+1$ are attributable to differences in accelerations of parents in generation $m$.

Lemma 4.2. Let $Y=\left(y_{0}, y_{1}, y_{2}, h\right)$ be a Riemannian scaled triple for the Euclidean metric on $\mathbb{R}^{n}$ where $y_{0}, y_{1}, y_{2}$ are not colinear. Then there is a sequence $\left\{\beta_{m}>0\right.$ : $m \geq 1\} \subset \mathbb{R}$ and, for each $m \geq 1$, a perturbation of the Euclidean metric to a Riemannian metric $\langle,\rangle_{m}$ on $\mathbb{R}^{n}$ with the following properties, where

$$
Y^{1}, Y^{2}, \ldots, Y^{2 m}
$$

are the descendants of the scaled triple $Y$ of $\left(\mathbb{R}^{n},\langle\rangle,\right)$ in generation $m$.

(a) $\langle,\rangle_{m}$ is $C^{\infty}$-close to Euclidean.

(b) $\langle,\rangle_{m}$ is Euclidean on the convex hull of the vertices of any $Y^{i}$ where $i=$ $1,2, \ldots, 2^{m}$.

(c) The vertices of $Y^{i}$ are not colinear for any $i=1,2, \ldots, 2^{m}$.

(d) Let $\alpha\left(Y^{j}\right)_{l}$ denote the parallel translation

$$
\alpha\left(Y^{j}\right)_{(2 j+1) h / 2^{m} \rightarrow(2 j+3) h / 2^{m}}
$$

of $\alpha\left(Y^{j}\right)$ along $p_{m}$ all calculated with respect to the Riemannian metric $\langle,\rangle_{l}$ where $l \geq m$. Then

$$
\left\|\alpha\left(Y^{j}\right)_{l}-\alpha\left(Y^{j+1}\right)\right\|>\beta_{m}
$$

for $j=1,2, \ldots, 2^{m}-1$ and all $l \geq m$.

(e) If $Y^{i}, Y^{j}$ have a common ancestor in generation $r<m$, then the norms of differences, after parallel translation along $p_{m}$, of $\alpha\left(Y^{i}\right), \alpha\left(Y^{j}\right)$ are smaller than $\beta_{r} / 4$ for the Riemannian metric $\langle,\rangle_{l}$ and any $l \geq m$.

(f) The sequence $\left\{\langle,\rangle_{m}: m \geq 1\right\}$ converges as a sequence of $C^{\infty}$ Riemannian metrics to a $C^{\infty}$ Riemannian metric $\langle,\rangle_{\infty}$. 
Proof. In Lemma 4.1 write $\beta_{1}=\left\|\alpha\left(Y^{l}\right)-\alpha\left(Y^{R}\right)\right\| / 2$. Set $\langle,\rangle_{1}=\langle$,$\rangle . Then since$ $\langle,\rangle_{1}$ is Euclidean on the convex hull of the vertices of $Y^{l}, Y^{R}$ condition (e) holds when $m=1$. The other conclusions depend on the $\beta_{m}$ where $m>1$ and we define these inductively as follows.

Suppose that Lemma 4.2 holds for $m<k$ and let

$$
Z^{1}, Z^{2}, \ldots, Z^{2^{k-1}}
$$

be the descendants of $Y$ in generation $k-1$. Apply Lemma 4.1 to each $Z^{j}$, choosing perturbations so small that for any $j=1,2, \ldots, 2^{k-1}$ the difference in norms of accelerations of any pair from

$$
Z^{j},\left(Z^{j}\right)^{L},\left(Z^{j}\right) R
$$

is less than

$$
\left(\min _{m=1,2, \ldots, k-1} \beta_{m}\right) / 2^{k+2} .
$$

Take care also that the perturbations are so small that they do not undo the previous inequalities (d) for $m \leq k-1$. Now let $W^{1}, W^{2}, \ldots, W^{2 k}$ be the immediate descendants of the $Z^{j}$ and set

$$
\beta_{k}=\min _{i=1,2, \ldots, 2^{k}-1}\left\|\alpha\left(W^{i}\right)-\alpha\left(W^{i+1}\right)\right\| / 2 .
$$

To ensure the convergence in (f) make each perturbation so much smaller than the last that $\left\{\langle,\rangle_{m}: m \geq 1\right\}$ is Cauchy. As in [3], Theorem 1.1.11, the $C^{\infty}$ Riemannian metrics comprise a complete metric space, which proves (f).

To prove Theorem 3 consider the perturbation $\langle,\rangle_{\infty}$ of the Euclidean metric and the associated $Y^{w}$ for words $w$ in $L, R$. If $s=2 i h / 2^{m} \in D_{m}$, then

$$
\left\|\alpha_{m-}(s)-\alpha_{m+}(s)\right\|>\beta_{m}
$$

by Lemma 4.2(d). By Lemma 4.2(e)

$$
\left\|\alpha_{m \pm}(s)-\alpha_{\infty \pm}(s)\right\| \leq \beta_{m} / 4
$$

and therefore

$$
\left\|\alpha_{\infty-}(s)-\alpha_{\infty+}(s)\right\| \geq \beta_{m} / 2 .
$$

Theorem 3 now follows from Lemma 3.1.

\section{ACKNowledgments}

The author thanks the referee for a thoughtful reading and constructive suggestions.

\section{REFERENCES}

1. A. S. Cavaretta, W. Dahmen and C. A. Micchelli, Stationary subdivision, Mem. Amer. Math. Soc. 93 (1991). MR 92h:65017

2. S. Gallot, D. Hulin and J. Lafontaine, Riemannian Geometry, Springer-Verlag, 1990. MR 91j:53001

3. R. Narasimhan, Analysis on real and complex manifolds, Advanced Studies in Pure Math. 1, Elsevier, 1973. MR 49:11576

4. G. de Rham, Sur quelques fonctions différentiables dont toutes les valeurs sont des valeurs critiques, Celebrazioni archimedee de secolo XX, Siracusa, Vol. II, 61-65, 11-16 aprile 1961. MR 27:1551 
5. __ Un peu de mathématiques á propos d'une courbe plane, Revue de Mathématiques Élémentaires 2 (1947), 73-76, 89-97. MR 9:246g

6. Sur certaines équations fonctionelles, Ouvrage publié á l'occasion de son centenaire par l'École polytechnique de l'Université de Lausanne, 95-97, 1953. MR 19:842d

7. __ Sur une courbe plane, J. de Math. Pures Appl. 35 (1956), 25-42. MR 19:842e

8. _ Sur les courbes limites de polygones obtenus par trisection, L'Enseignement Mathématique 5 (1959), 29-43. MR 21:7508

9. L. Noakes, Asymptotically smooth splines, Advances in Computational Math. 4 (1994), 131137. MR 96h:65016

10. _ A global algorithm for geodesics, to appear in Bull. Austral. Math. Soc. Series A.

11. _ Riemannian quadratics, submitted to Proc. Third Chamonix Conference on Curves and Surfaces, A. Le Méhauté, C. Rabut and L. L. Schumaker (eds.) (1997), 319-328.

12. H. von Koch, Sur une courbe continue sans tangente obtenue par une construction géométrique élémentaire, Arkiv. Mat. Astronomik Fysik 1 (1904), 681-702.

Department of Mathematics, The University of Western Australia, Nedlands, Western Australia 6907, Australia

E-mail address: lyle@maths.uwa.edu.au 